\title{
Spectroscopic Evidence for Quasar Microlensing
}

\author{
L. Wisotzki ${ }^{1}$, S. Lopez ${ }^{2}$, O. Wucknitz ${ }^{3}$ \\ ${ }^{1}$ Astrophysical Institute Potsdam, An der Sternwarte 16, D-14482 Potsdam, Germany, \\ email: lwisotzki@aip.de \\ ${ }^{2}$ Departamento de Astronomía, Universidad de Chile, Casilla 36-D, Santiago, Chile \\ ${ }^{3}$ Institut für Physik, Universität Potsdam, Am Neuen Palais 10, D-14469 Potsdam
}

\begin{abstract}
We report on a number of ongoing experiments to obtain spectrophotometric data of lensed quasars. We find that spectral differences are a widespread phenomenon, suggesting that microlensing is very common. We discuss some aspects of how to distinguish microlensing from other sources of spectral differences. Extinction by intervening dust can have similar broad band signatures, but can be distinguished or even separated from microlensing using spectrophotometry. We present recent results on extinction and microlensing in a $z=0.93$ lensing galaxy. We also present a case study of the issue of discrepant flux ratios in a quadruple QSO, where we find that microlensing probably plays a role. We finally discuss the aspect of microlensing-induced emission line profile variations.
\end{abstract}

\section{Introduction}

Microlensing is expected to be a common phenomenon among multiply imaged quasars, at least if the lenses contain sufficient mass density in the form of stellar and other compact objects. The standard observational approach to microlensing by photometric monitoring is however expensive, and solid evidence for or against microlensing being important does not yet exist for many sources. But microlensing can also influence quasar spectra, and the spectroscopic comparison between lensed quasar components can be used as an additional test for the action of microlensing.

Although microlensing in itself is achromatic, the amount of magnification depends on the source size if the latter is comparable with or larger than the Einstein radius $\theta_{E}$ of microlenses. Assuming $\theta_{E}$ to be typical for low-mass stars, the following phenomena can be expected in the spectra of quasars: (i) The optical/UV continuum emitting region has a size presumably $R_{c} \lesssim \theta_{E}$. It can therefore get highly magnified. (ii) The size of the broad emission line region (BLR) has been measured in several low- $z$ quasars by reverberation mapping (e.g., Kaspi et al. 2000). Typical values are $R_{\mathrm{BLR}} \sim 0.01-0.1 \mathrm{pc}$ which is $\gg R_{c}$, thus the flux amplification of broad emission lines will in general be small. We therefore expect that microlensing can affect the line/continuum ratio. If a caustic moves over certain regions of the BLR, also the line profile may be altered. (iii) For an accretion disk with temperature stratification (such as the 'standard' thin disk), the effective size varies with wavelength. The quasar continuum source looks bigger at longer wavelengths, and this may lead to 'chromatic' microlensing.

All these effects are in principle observable in single-epoch data. Multiple epochs are nevertheless required to safely distinguish between intrinsic variability compounded with time-delay effects from microlensing-induced spectral differences. In this paper we report on a number of ongoing experiments to obtain spectrophotometric data of lensed quasars. 


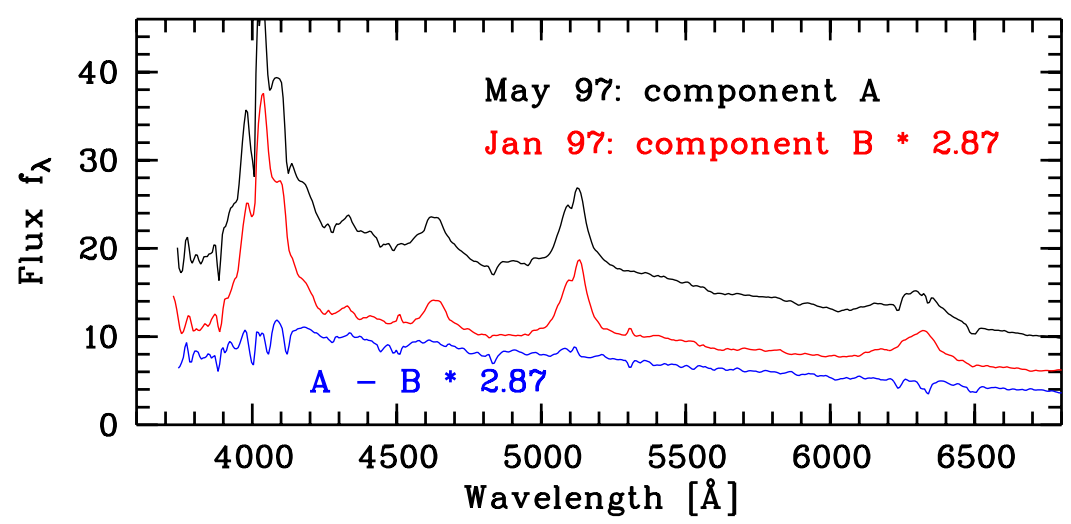

Figure 1. Spectrophotometric comparison of the two components of the double QSO HE 1104-1805, with the time delay taken out. The spectral differences can only be due to microlensing.

\section{HE 1104-1805}

In 1993 we discovered the double QSO HE 1104-1805 and found the spectra of components A and B to be distinctly different (Wisotzki et al. 1993), essentially displaying all the above mentioned phenomena (except line profile variations). We conducted several repeat spectrophotometric observations between 1993 and 2003, including a period of $\sim$ monthly monitoring in 1995-1998. An example set of spectra is shown in Fig. 1, where $\mathrm{A}$ and $\mathrm{B}$ are displayed at different epochs, eliminating the time delay. While the emission line ratio A/B is consistenly 2.87 at essentially all epochs, component A shows an additional continuum excess that makes this component significantly bluer in the continuum than B. Most likely, this additional component is due to selective continuum amplification in component A which is located closer to the centre of the lensing galaxy. Optical photometric monitoring by the OGLE project (Schechter et al. 2003) has now also shown that there is definitely strong microlensing going on in component A (and much less so in B). HE 1104-1805 is probably the clearest known case of spectroscopic microlensing and can be used a template for further investigations in other sources.

Table 1. Summary of spectroscopic microlensing diagnostics for our sample. We list whether the object shows evidence for selective continuum amplification, and whether there are chromatic differences between the components.

\begin{tabular}{|c|c|c|c|}
\hline Object & line/cont ML & chromatic ML & Ref. \\
\hline HE $0047-1756$ & probably & probably & Wisotzki et al. (2004b) \\
\hline $\begin{array}{l}\text { HE } 0142-1000=\text { UM } 673 \\
\text { HE } 0156-4329=\text { CTQ } 414\end{array}$ & $\begin{array}{l}\text { probably not } \\
\text { unknown }\end{array}$ & no & Wisotzki et al. (2004a) \\
\hline HE $0230-2130$ & probably & unknown & Wisotzki et al. (1999) \\
\hline HE $0435-1223$ & yes & weak? & Wisotzki et al. (2003) \\
\hline HE $0512-3329$ & yes & yes & Wucknitz et al. (2003) \\
\hline HE 1104-1805 & yes & yes & Wisotzki et al. (1993) \\
\hline HE $1352-2242=$ CTQ 327 & probably & no & Morgan et al. (2003) \\
\hline HE 2149-2745 & yes & yes & Burud et al. (2002) \\
\hline
\end{tabular}




\section{Microlensing in a complete sample of lensed QSOs}

Over the last years we have collected spectrophotometric data on several lensed QSOs, many of them discovered (some also rediscovered) in the course of the Hamburg/ESO survey for bright QSOs (Wisotzki et al. 2000). Just considering the HES-selected objects, our sample is very nearly complete. A summary of the observational results is provided in Table 1. Remarkably, we find that spectral differences of the type detected in HE 1104-1805 are present in many other QSOs as well. In particular, the line-tocontinuum ratio is different in almost every case, whereas a smaller number of objects shows also indications for a chromatic effect. For some objects the differences are obvious: Besides HE 1104-1805, the double QSOs HE 0047-1756 and HE 0512-3329 show very similar phenomena. In others the effects are weaker and only detectable at good signal to noise ratio. All objects where only single-epoch observations are available have been marked as at most 'probable'. Although time delays are unknown for most objects in the sample, the occurence of a similar phenomenon in repeat observations is very suggestive of microlensing rather than intrinsic variability. Taken together, there is a remarkably high occurence of spectra differences, suggesting that microlensing in multiply imaged QSOs is a widespread phenomenon.

Since observations of lensed QSOs are much more frequently conducted by broad band imaging, e.g. in the context of monitoring programmes, we want to add a cautionary note. Broad band colours of QSOs may be heavily affected by a varying emission line to continuum contrast when strong emission lines are located inside the band. In fact, the A component of HE 1104-1805 has a bluer continuum slope than component B, but it is 'redder' in $B-V$ simply because of emission line contamination. Every attempt to detect chromatic differences, or to assess extinction, should make sure that this effect plays no role.

\section{Spectral differences: Microlensing vs. extinction}

Another process that can cause spectral differences in lensed QSOs are differences in the intervening extinction between the two lines of sight. Broad band colours alone are insufficient to distinguish between extinction and microlensing, but since extinction acts on the continuum and line emission alike, the two effects can be separated spectroscopically. Our HST/STIS spectra of the close double HE $0512-3329\left(z_{\mathrm{Q}}=1.58\right)$ exhibit

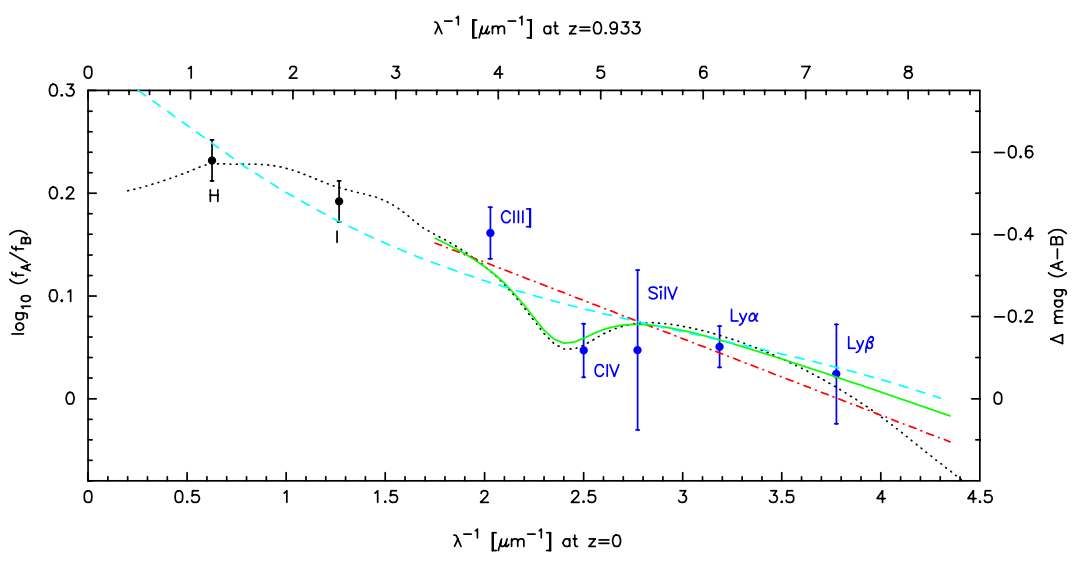

Figure 2. Extinction curve of the intervening galaxy at $z=0.93$ in HE 0512-3329. The points are measurements, the curves show various model fits (from Wucknitz et al. 2003). 
clearly different spectral slopes in $\mathrm{A}$ and $\mathrm{B}$, but the continuum and emission line ratios A/B are not compatible (Wucknitz et al. 2003). The continuum flux ratio can thus not be used to get an extinction curve; only the emission lines. In Fig. 2 we show that the extinction curve is nevertheless quite well constrained, favouring in fact a Galactic curve with $2200 \AA$ bump. After applying the extinction correction to the continuum flux ratio, we arrive at the pure microlensing signal (Fig. 3) acting on the continuum source.

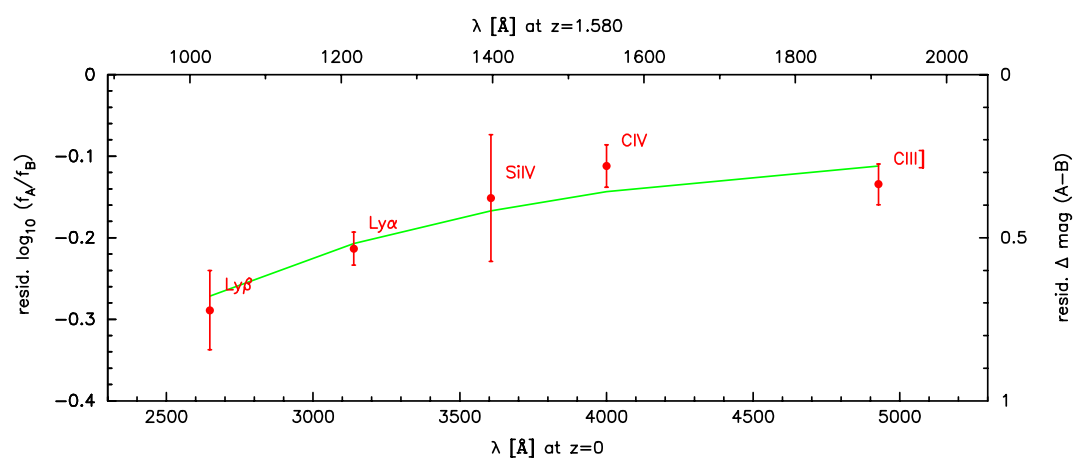

Figure 3. Microlensing amplification ratio A/B in HE 0512-3329 after correcting for extinction. The points correspond to the detected emission lines (from Wucknitz et al. 2003).

\section{Flux ratios of lensed QSOs}

If the BLR is indeed much bigger than the microlensing scale, then the emission line fluxes should mirror the 'true' flux ratios of the components, i.e. those given by macrolensing of the smooth galaxy potential alone (provided that extinction is negligible). This is interesting in the light of the recent discussion about the fact that simple mass models often poorly reproduce the (continuum) flux ratios in multiply imaged quasars. We have investigated this issue in the new quadruple system HE 0435-1223 using the PMAS integral field spectrophotometer on the Calar Alto $3.5 \mathrm{~m}$ telescope. We find that the match between models and continuum flux ratios is poor, and that the emission line flux ratios are significantly closer to the model, although the discrepancies are still substantial. Table 2 documents our measurements. In this object, microlensing apparently contributes to the discrepant flux ratios but it is probably not the whole story.

Table 2. Observed and predicted flux ratios between the four components of HE 0435-1223: (1) $\mathrm{C}$ IV and C III] emission lines; (2) continuum ratios at $\lambda=4580 \AA$; (3) broad-band measurement; (4) flux ratios predicted by a simple mass model (from Wisotzki et al. 2003)

\begin{tabular}{lrrrr}
\hline Ratio & lines continuum & $g$ band & Model \\
& & & & \\
\hline B/A & 0.77 & 0.62 & 0.60 & 1.11 \\
C/A & 0.71 & 0.54 & 0.57 & 1.04 \\
D/A & 0.56 & 0.61 & 0.55 & 0.68 \\
C/B & 0.93 & 0.87 & 0.95 & 0.94 \\
D/B & 0.73 & 0.98 & 0.93 & 0.61 \\
D/C & 0.79 & 1.12 & 0.97 & 0.65 \\
\hline
\end{tabular}




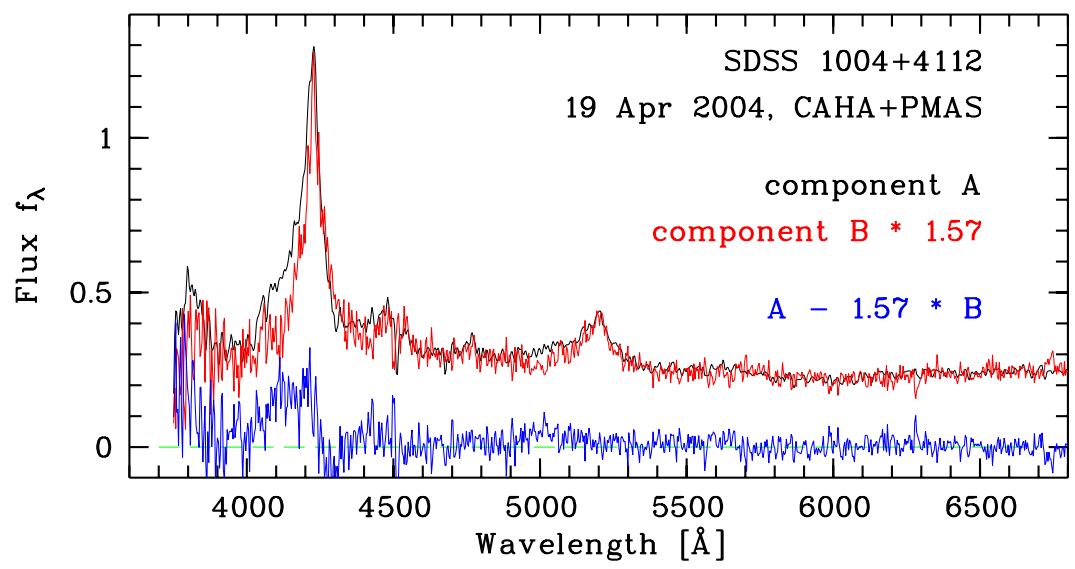

Figure 4. PMAS spectra of the A and B components of the wide-separation quadruple QSO SDSS 1004+4112. The C IV blue wing discrepancy found by Richards et al. (2004) persists, but the continua are absolutely identical.

\section{Microlensing of the broad line region?}

We have also compared the emission line profiles in the spectra of our sample. The only object where we find statistically significant profile differences is HE 1104-1805 (at certain epochs only; for most epochs the profiles are identical even at a $\mathrm{S} / \mathrm{N}$ of 100 in A). However, even this was false alarm: The differences in C IV profile disappear completely when comparing spectra of A and B such that the time delay is eliminated. Apparently, the variations were intrinsic to the QSO.

The observed lack of line profile differences in single-epoch data places interesting constraints on (i) the amplitude of intrinsic line shape variations (must be small, or one would see more differences) and (ii) the occurence of microlensing-induced line shape variations (must also be small).

Recently, Richards et al. reported highly significant emission line discrepancies between the A and B components of the wide-separation quadruple system SDSS 1004+4112 and suggested microlensing as an explanation. We observed the A and B components with PMAS on the Calar Alto $3.5 \mathrm{~m}$ telescope in April 2004. The extracted spectra are shown in Fig. 4. We find almost exactly the same differences in the C IV line profile as seen in the May 2003 data by Richards et al. (which are however not present in their Nov-Dec data). While Richards et al. could not measure the continuum shapes reliably, PMAS is an integral field spectrophotometer with essentially zero slit losses, and we can therefore also measure the continuum ratio $\mathrm{A} / \mathrm{B}$. It is, to the limits of measurement accuracy, identical over the whole spectral range. If the microlensing hypothesis of Richards et al. is correct, then microlensing acts only on a selected group of BLR clouds, not at all on the continuum, and the same situation occured at least twice, in May 2003 and in May 2004. While this sounds contrived, we have no better explanation to offer, at present. Clearly further spectrophotometric observations of this source are needed.

\section{Conclusions}

Our collected spectrophotometric database indicated that spectral differences between multiply imaged quasars are the rule rather than the exception. Consequently, a significant fraction of the surface mass density in the lensing galaxies must be in the form of compact objects. This has several practical consequences: 
- Colour differences between multiply imaged QSOs cannot be interpreted as being purely due to extinction per default.

- Broad band colour differences can be influenced by line to continuum ratios.

- Spectrophotometry can lift the degeneracy between microlensing and extinction. There is no evidence that emission line profile variations caused by microlensing is a common phenomenon. The only example where such an effect may be at work (SDSS 1004+4112) appears to be quite difficult to understand.

\section{Acknowledgements}

We acknowledge several allocations of observing time on HST, Magellan, Calar Alto, and ESO telescopes. DFG travel grants under Wi 1369 enabled the observations. This work was partially supported by the DLR Verbundforschung under 50 OR 0208. SL acknowledges support from the Chilean Centro de Astrofísica FONDAP No. 15010003, and from FONDECYT grant Nº3000 001.

\section{References}

Burud, I., Courbin, F., Magain, P., et al. 2002, A\&A, 383, 71

Gregg, M. D., Wisotzki, L., Becker, R. H., et al. 2000, AJ, 119, 2535

Kaspi, S., Smith, P. S., Netzer, H., et al. 2000, ApJ, 533, 631

Morgan, N. D., Gregg, M. D., Wisotzki, L., et al. 2003, AJ, 126, 696

Schechter, P. L., Udalski, A., Szymański, M., et al. 2003, ApJ, 584, 657

Wisotzki, L., Köhler, T., Kayser, R., Reimers, D. 1993, A\&A, 278, L15

Wisotzki, L., Christlieb, N., Liu, M., et al. 1999, A\&A, 348, L41

Wisotzki, L., Christlieb, N., Bade, N., et al. 2000, A\&A, 358, 77

Wisotzki, L., Schechter, P. L., Bradt, H. V., Heinmüller, J., \& Reimers, D. 2002, A\&A, 395, 17

Wisotzki, L., Becker, T., Christensen, L., et al. 2003, A\&A, 408, 455

Wisotzki, L., Becker, T., Christensen, L., et al. 2004a, AN, 325, 135

Wisotzki, L., Schechter, P.L., Chen, H.-W., et al. 2004b, A\&A, 419, L31

Wucknitz, O., Wisotzki, L., Lopez, S., \& Gregg, M. D. 2003, A\&A, 405, 445 